\title{
Oxygen-vacancy driven electron localization and itinerancy in rutile-based $\mathrm{TiO}_{2}$
}

\author{
Frank Lechermann, ${ }^{1,2}$ Wolfgang Heckel, ${ }^{2}$ Oleg Kristanovski, ${ }^{1}$ and Stefan Müller ${ }^{2}$ \\ ${ }^{1}$ I. Institut für Theoretische Physik, Universität Hamburg, D-20355 Hamburg, Germany \\ ${ }^{2}$ Institut für Keramische Hochleistungswerkstoffe, \\ Technische Universität Hamburg-Harburg, D-21073 Hamburg, Germany
}

\begin{abstract}
Oxygen-deficient $\mathrm{TiO}_{2}$ in the rutile structure as well as the $\mathrm{Ti}_{3} \mathrm{O}_{5}$ Magnéli phase is investigated within the charge self-consistent combination of density functional theory (DFT) with dynamical mean-field theory (DMFT). It is shown that an isolated oxygen vacancy $\left(\mathrm{V}_{\mathrm{O}}\right)$ in titanium dioxide is not sufficient to metallize the system at low temperatures. In a semiconducting phase, an ingap state is identified at $\varepsilon_{\mathrm{IG}} \sim-0.75 \mathrm{eV}$ in excellent agreement with experimental data. Bandlike impurity levels, resulting from a threefold $\mathrm{V}_{\mathrm{O}}-\mathrm{Ti}$ coordination as well as entangled $\left(t_{2 g}, e_{g}\right)$ states, become localized due to site-dependent electronic correlations. Charge localization and strong orbital polarization occur in the $\mathrm{V}_{\mathrm{O}}$-near $\mathrm{Ti}$ ions, which details can be modified by a variation of the correlated subspace. At higher oxygen vacancy concentration, a correlated metal is stabilized in the Magnéli phase. A $V_{O}$-defect rutile structure of identical stoichiometry shows key differences in the orbital-resolved character and the spectral properties. Charge disproportionation is vital in the oxygen-deficient compounds, but obvious metal-insulator transitions driven or sustained by charge order are not identified.
\end{abstract}

\section{INTRODUCTION}

From two motivating research directions, the investigation of oxygen-deficient transition-metal oxides has gained enormous renewed interest. First, the emerging field of oxide heterostructures lead to questions concerning the impact of oxygen vacancies on interface properties. Since especially the $\mathrm{SrTiO}_{3}$ band insulator marks an important heterostructure building block, elucidating the role of such vacancies in that compound has recently attracted lots of attention. 1 7 Second, on the search of realizing a memristor $\frac{8}{8} \mathrm{TiO}_{2-\delta}$ remains a key material. ${ }^{[10}$ Formation and migration of oxygen-vacancy defects are identified to regulate the resistance modulation therein.

Stoichiometric $\mathrm{SrTiO}_{3}$ is characterized as a cubic (perovskite) $\mathrm{Ti}^{4+}\left(3 d^{0}\right)$ compound with crystal-field split $e_{g}$ and $t_{2 g}$ states (cf. Fig. 11). The band gap is located between the dominantly $\mathrm{O}(2 p)$ - and the $t_{2 g}$ manifold. Due to the strong $\mathrm{O}(2 p)-e_{g}$ hybridization, the creation of an oxygen vacancy $\left(\mathrm{V}_{\mathrm{O}}\right)$ leads to local $\mathrm{Ti}^{3+}\left(3 d^{1}\right)$ sites and $e_{g}$-dominated in-gap states 11 The interplay of $\mathrm{Ti}^{4+}$ and $\mathrm{Ti}^{3+}$-like states gives rise to a competition between electron localization and itinerancy, posing an intriguing many-body problem. Recently, that problem was approached by theory within the combination of density functional theory (DFT) with dynamical mean-field theory (DMFT) ${ }^{[5|6| 11}$ Experiments indeed suggest that $\mathrm{V}_{\mathrm{OS}}$ on the surface of strontium titanate as well as in the interface of $\mathrm{LaAlO}_{3} / \mathrm{SrTiO}_{3}$ may be relevant not only for metallicity, but also for emergent magnetic and/or superconducting order.

Oxygen vacancies in $\mathrm{TiO}_{2}$ pose a related intricate problem, yet with a twist. Besides single-defect scenarios, long-range-ordered vacancy structures provided by the $\mathrm{Ti}_{n} \mathrm{O}_{2 n-1}$ Magnéli phases $\frac{12}{14}$ are an additional point of materials reference. The role of oxygen vacancies in titanium dioxide, with its twofold structural representations of rutile and anatase is a longstanding problem, and has so far been studied in several theoretical works based on conventional DFT, ${ }^{15}$ using hybrid functionals, $\frac{16}{20}$ as well as by treating static electronic correlations within DFT + U 20 24 Within the latter framework, Mattioli et al. ${ }^{21}$ originally showed that isolated $\mathrm{V}_{\mathrm{O}}$ in $\mathrm{TiO}_{2}$ can introduce shallow electronic levels only in anatase, while solely deep localized levels are induced in rutile. The more recent oxygen-deficient rutile studies $\frac{18|22| 24}{\text { suggest }}$ an intricate coexistence of shallow and deep levels.

Electronic structure investigations of various Magnéli phases, which may in fact be derived starting from the rutile structure 15 furthermore revealed challenging physics, such as e.g. metal-insulator transitions and charge ordering! ${ }^{25}$ But those assessments are so far limited by the possibilities of $\mathrm{DFT}(+\mathrm{U})$ to describe electron correlation.

In this work we want to provide a theoretical account of electron correlations in oxygen-deficient rutile-based $\mathrm{TiO}_{2}$ from a DFT+DMFT perspective. This not only provides a relevant examination of defect-mediated electronic self-energy effects beyond Kohn-Sham exchangecorrelation treatments for an oxide compound with high potential for technological applications. It also allows

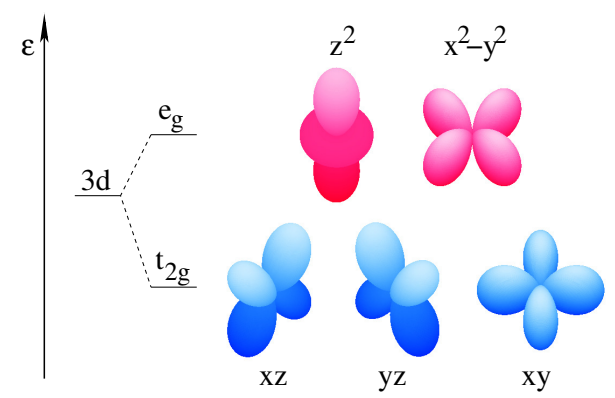

FIG. 1. (color online) Crystal-field splitting of a transitionmetal $3 d$ shell, located in a full-cubic symmetry environment, into $t_{2 g}$ and $e_{g}$ states. 
us to compare the characteristics of the induced defect states to e.g. the ones found in the $\mathrm{SrTiO}_{3}$ perovskite. We show that energy-similar in-gap states are emerging upon creation of $\mathrm{V}_{\mathrm{O}} \mathrm{s}$, but metallicity does only occur above a corresponding concentration threshold.

Rutile is known to be the thermodynamically stable $\mathrm{TiO}_{2}$ phase at all temperatures and pressures, 28 while anatase is metastable but can kinetically be stabilised at low temperatures. We therefore restrict the investigation on the rutile structural case of $\mathrm{TiO}_{2}$ as well as the $\mathrm{Ti}_{3} \mathrm{O}_{5}$ Magnéli phase as a higher $\mathrm{V}_{\mathrm{O}}$-concentration counterpart.

\section{COMPUTATIONAL APPROACH}

The supercell defect structures of rutile- $\mathrm{TiO}_{2-\delta}$ as well as the Magnéli $\mathrm{Ti}_{3} \mathrm{O}_{5}$ structure are structurally relaxed $\underline{10}$ on the DFT level within the generalized-gradient approximation (GGA) using the PBEsol ${ }^{29}$ functional in the VASP code 30

Our present charge self-consistent DFT+DMFT framework ${ }^{34}$ builds up on the mixed-basis pseudopotential approach $\frac{37 / 38}{}$ for the DFT part and the continuous-time quantum-Monte-Carlo method $39 \sqrt{30}$, as implemented in the TRIQS package, ${ }^{4142}$ for the DMFT impurity problem. We utilize the GGA in the PBE functional form within the Kohn-Sham cycle.

Locally, threefold effective $\operatorname{Ti}(3 d)$ functions define the correlated subspace, which as a whole consists of the corresponding sum over the various $\mathrm{Ti}$ sites in the defect problem. Projected-local orbitals 44 of $3 d$ character provide the effective functions from acting on Kohn-Sham conduction states above the $\mathrm{O}(2 p)$-dominated band manifold. Note that the resulting effective orbitals are not of exclusive $t_{2 g^{-}}$or $e_{g}$ kind, but are defined by the local three-orbital sector lowest in energy, respectively. Each Ti site marks an impurity problem, and the whole number of explicitly treated impurity problems depends on the number of symmetry-inequivalent transition-metal sites in the given supercell. A three-orbital Hubbard Hamiltonian of Slater-Kanamori form, if not otherwise stated parametrized by the Hubbard $U=5 \mathrm{eV}$ and the Hund's exchange $J_{\mathrm{H}}=0.7 \mathrm{eV}$, is active on each Ti site. These values for the local Coulomb interactions are in line with previous studies on titanates.492 A doublecounting correction of the fully-localized form $\frac{53}{5}$ is utilized in this work. The analytical continuation of the finite-temperature Green's functions on the Matsubara axis $i \omega$ to real frequencies is performed via the maximumentropy method.

Note that we study only paramagnetic states without broken spin symmetry, as well as charge-neutral lattice configurations. Yet possible inter-site charge disproportionation is surely allowed.

\section{RESULTS}

\section{A. Rutile $\mathrm{TiO}_{2}$}

To set the stage for the discussion of oxygen-deficient titanium dioxide, we briefly discuss the electronic structure at stoichiometry. The rutile structure ${ }^{54}$ (see Fig. 2a) has tetragonal symmetry (space group $P 4_{2} / \mathrm{mnm}$ ) with a ratio $c / a=0.64$ and the primitive cell comprises two $\mathrm{TiO}_{2}$ formula units. It consistes of corner- and edgesharing $\mathrm{TiO}_{6}$ octahedra, such that each oxygen ion is coordinated by three neighboring titanium ions. This is in contrast to common perovskite-based titanates, where the $\mathrm{TiO}_{6}$ octahedra are exclusively corner-sharing and $\mathrm{O}$ is twofold Ti-coordinated. Of the three rutile minimal Ti-O bond lengths, the two shorter ones are identical in extent. Nominally, titanium is in the $\mathrm{Ti}^{4+}$ oxidation state with $3 d^{0}$ occupation.

The given compound is a band insulator with an experimental (optical) band gap of size $\Delta_{\mathrm{g}} \sim 3 \mathrm{eV}$ ventional DFT calculations yield a smaller gap $\Delta_{\mathrm{g}} \sim 2 \mathrm{eV}$ (cf. Fig. 2b). Since $\mathrm{TiO}_{2}$ is a nominal $3 d^{0}$ material, the HOMO orbitals are of dominant $\mathrm{O}(2 p)$ kind and the LUMO orbitals are mainly of $\operatorname{Ti}\left(3 d_{t_{2 g}}\right)$ character, while dominant $\operatorname{Ti}\left(3 d_{e_{g}}\right)$ is located at even higher energies above the band gap. Because of the fact that the $c$-axis and the main $\mathrm{TiO}_{6}$-octahedra axes are locally
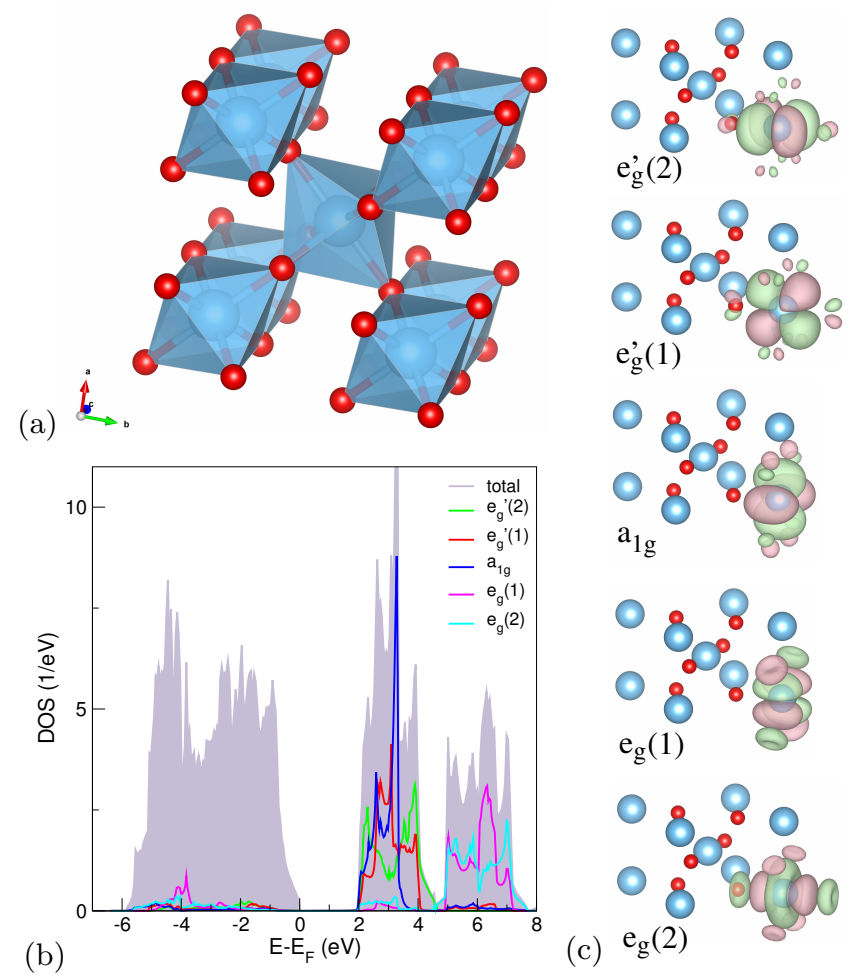

FIG. 2. (color online) Characterization of rutile- $\mathrm{TiO}_{2}$. (a) Crystal structure with $\mathrm{Ti}$ (large blue/grey) and $\mathrm{O}$ (small red/dark) atoms. (b) Total and local-orbital GGA density of states. (c) Projected local $\mathrm{Ti}(3 d)$ orbitals. 


\begin{tabular}{l|cccccc}
\hline \hline orbital & $\varepsilon_{\mathrm{CF}}$ & $\left|z^{2}\right\rangle$ & $|x z\rangle$ & $|y z\rangle$ & $\left|x^{2}-y^{2}\right\rangle$ & $|x y\rangle$ \\
\hline$\left|e_{g}^{\prime}(2)\right\rangle$ & 2684 & 0.000 & 0.000 & 0.000 & 1.000 & 0.000 \\
$\left|e_{g}^{\prime}(1)\right\rangle$ & 2653 & 0.000 & 0.707 & 0.707 & 0.000 & 0.000 \\
$\left|a_{1 g}\right\rangle$ & 2574 & -0.827 & 0.000 & 0.000 & 0.000 & -0.562 \\
$\left|e_{g}(1)\right\rangle$ & 4398 & 0.000 & -0.707 & 0.707 & 0.000 & 0.000 \\
$\left|e_{g}(2)\right\rangle$ & 4413 & 0.562 & 0.000 & 0.000 & 0.000 & -0.827 \\
\hline \hline
\end{tabular}

TABLE I. The titanium $t_{2 g}=\left(e_{g}^{\prime}(2), e_{g}^{\prime}(1), a_{1 g}\right)$ and $e_{g}=$ $\left(e_{g}(1), e_{g}(2)\right)$ orbitals in $\mathrm{TiO}_{2}$ with their respective crystalfield level $\varepsilon_{\mathrm{CF}}$ (in meV), expressed in terms of cubic harmonics.

aligned trigonal, the $\operatorname{Ti}\left(t_{2 g}, e_{g}\right)$ orbitals may be written as linear combinations of cubic harmonics from diagonalization of the orbital-density matrix (see Tab. I). Due to the local symmetry, the internal $\left(t_{2 g}, e_{g}\right)$ degneracies known from the full octahedral group are lifted, respectively. Comparison of the crystal-field levels $\varepsilon_{\mathrm{CF}}$ marks the $a_{1 g}$ level as the lowest one, $110 \mathrm{meV}$ below $e_{g}^{\prime}(2)$. The $t_{2 g}$-based states are about $2.2 \mathrm{eV}$ lower in energy than the $e_{g}$-based ones. Within the effective $t_{2 g}$ manifold, which has a bandwdith of about $2.5 \mathrm{eV}$, the $e_{g}^{\prime}(2)$ orbital is designated since its lobes point along the in-plane tetragonal axes (see Fig. 2f).

\section{B. Oxygen vacancy in rutile $\mathrm{TiO}_{2}$}

\section{Structural details and correlated subspaces}

As shown in Fig. 3 a supercell five times the size of the primitive cell, i.e. with $10 \mathrm{Ti}$ and $20 \mathrm{O}$ atoms, serves as basis structure for the defect study. A single $\mathrm{V}_{\mathrm{O}}$ leaves three nearest-neighbor titanium ions behind, here labelled Ti1, Ti2 and Ti3. In the stoichiometric rutile structure, the Ti2-Ti3 distance marks the short side of the given Ti triangle. The nominal $\mathrm{V}_{\mathrm{O}}$ concentration in this constellation amounts to $c \equiv \delta / 2=0.05$, i.e. our modeling describes a $\mathrm{TiO}_{1.9}$ defect case. This represents a large $V_{O}$ concentration, however not unrealistic for the given system.58 Table [I] shows that the inter-atomic distances are only weakly modified upon structural relaxation, which may be also related to the small supercell size. Still, the obtained pattern describes a shortening of the Ti1-Ti2,3 distances and an elongation of the Ti2Ti3 bonding, providing a trend to balance the triangle distances trough the $\mathrm{V}_{\mathrm{O}}$.

In the following we want to investigate the effect of an $\mathrm{V}_{\mathrm{O}}$ in the rutile structure in terms of the local-orbital configuration as well as the net electronic structure. We will discuss two different choices for the correlated subspace. First in section III B 2, that space is formed by all Ti sites in the given structure, which marks the canonical and ground-state-oriented case. Second in section IIIB 3. the correlated subspace is restricted to the contribution of the Ti1-3 sites, i.e. it becomes more local. This second

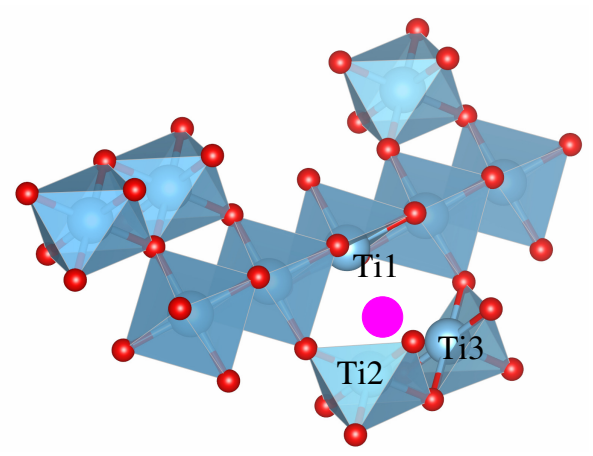

FIG. 3. (color online) Structural characterization of an oxygen vacancy (large purple/lightgrey) in rutile.

\begin{tabular}{l|ccc}
\hline & $\mathrm{Ti} 1-\mathrm{Ti} 2$ & $\mathrm{Ti} 1-\mathrm{Ti} 3$ & $\mathrm{Ti2}-\mathrm{Ti} 3$ \\
\hline $\mathrm{TiO}_{2}$ & 3.57 & 3.57 & 2.96 \\
$\mathrm{TiO}_{1.9}$ & 3.53 & 3.53 & 2.97 \\
\hline \hline
\end{tabular}

TABLE II. Comparison of the inter-atomic distances (in $\AA$ ) between $\mathrm{Ti}$ ions surroundig a vacancy-designated oxygen site.

choice may be interpreted as treating an excited state of the system, where electrons do not see the explicit Coulomb repulsion on the remaining Ti sites. Since the $3 d$ occupation on the original $\operatorname{Ti}\left(d^{0}\right)$ sites distant to the $\mathrm{V}_{\mathrm{O}}$ is expected small, double occupation is there rather rare. If moreover the electrons have gained energy from an excitation process, they can even more easily escape from such double occupations (as well as more efficiently screen the Coulomb penalties). Thus the average effect of local $U$ and $J_{\mathrm{H}}$ on the $\mathrm{V}_{\mathrm{O}}$-distant $\mathrm{Ti}$ sites can then be neglected to a good approximation to obtain a rough picture of the present system on a globally-higher excitation level. This approximation and interpretation is also not in conflict with the definition of a one-particle spectral function. We thus simply term that space 'excited correlated subspace', and this second choice allows us to shed light on possible changes in the oxidation state of the oxygen vacancy. Still note however that the Coulomb interactions on the Ti sites away from the $\mathrm{V}_{\mathrm{O}}$ are crucial to understand the semiconducting character of $\mathrm{TiO}_{2-\delta}$, as will be explained in the following section IIIB2,

\section{Canonical correlated subspace formed by all Ti sites}

Let us start with the well-defined DFT+DMFT setting of the correlated subspace build by all the Ti sites. On the GGA level, as displayed by plotting the total spectral function $A(\omega)=\sum_{\mathbf{k}} A(\mathbf{k}, \omega)$ in Fig. 4, the considered system becomes metallic with semimetallic tendencies around the Fermi level $\varepsilon_{\mathrm{F}}$ positioned within the $t_{2 g}$ manifold. Yet correlations and finite temperature $T$ render the situation more intriguing. At low $T$ the 


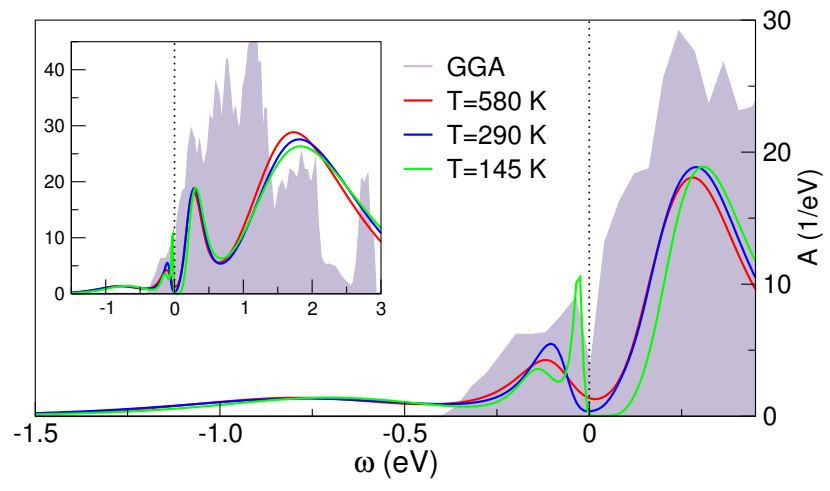

FIG. 4. (color online) Total spectral information for rutile$\mathrm{TiO}_{1.9}$ in GGA and DFT+DMFT. at different temperatures in two energy windows.

defect structure is semiconducting within DFT+DMFT, with a small charge gap $\Delta \sim 0.06 \mathrm{eV}$ at $T=145 \mathrm{~K}$. That gap is filled with rising temperature, marking a bad-metal regime. In addition, there is sizable transfer of spectral weight to a broad in-gap structure centered at $\varepsilon_{\mathrm{IG}} \sim-0.75 \mathrm{eV}$. Note that optics measurements detect an absorption peak in reduced semiconducting $\mathrm{TiO}_{2}$ single crystals at $0.75 \mathrm{eV}, \frac{55156}{5}$ commonly used to explain its blue color. ${ }^{58}$ Photoemission measurements on the rutile surface report a defect-state peak at $\sim-0.9 \mathrm{eV}, \underline{59}$ while on the anatase surface it is located at an higher energy of $\sim-1.1 \mathrm{eV} \sqrt{59 \mid 62]}$ Furthermore, scanning tunneling spectroscopy finds an in-gap state at $\sim-0.7 \mathrm{eV}$ on the defect-rutile surface ${ }^{63}$ and x-ray photoelectron spectroscopy on rutile $\mathrm{TiO}_{2}$ nanoparticles reveals a defect state at $\sim-0.8 \mathrm{eV} \stackrel{201}{20}$ it is tempting to relate these experimental findings of deep levels in $\mathrm{TiO}_{2-\delta}$ to the present satellite peak. On the other hand, $n$-type conductivity with rather high mobilities due to shallow level has been also reported in the literature, ${ }^{64}$ which might be connected to our small-gap feature.

To gain insight in the nature and characteristics of the Ti-local states near the $\mathrm{V}_{\mathrm{O}}$, first Tab. III provides the effective orbitals on Ti1-3 written in terms of linear combinations of the original $\left(t_{2 g}, e_{g}\right)$ functions from Tab. If The $\varphi$ and $\varphi^{\prime}$ orbitals on Ti2,3 behave very similarly, therefore we restrict the discussion to the $\varphi$ branch. It is seen that while the $\psi_{2}$ orbital has strong $e_{g}$ character, the orbitals $\varphi_{1}, \varphi_{2}$ have sizable contributions from both original orbital sectors. Thereby $\varphi_{1}$ is $t_{2 g}$-dominated and $\varphi_{2}$ is $e_{g}$-dominated. Thus a nearly exclusive $e_{g}$ character of the local defect states, as e.g. given in oxygen-deficient $\mathrm{SrTiO}_{3}, \frac{116}{16}$ does not apply for oxygen vacancies in $\mathrm{TiO}_{2}$. Note furthermore that the $e_{g}^{\prime}(2)$ orbital has almost negligible contribution to the $\mathrm{V}_{\mathrm{O}}$-induced physics, as the $e_{g}^{\prime}(2)$-dominated $\varphi_{3}$ orbital remains nearly empty.

Concerning tight-binding parameters, the hopping between both $\varphi_{1}$ on Ti2 and Ti3 is largest with $t_{\varphi_{1}}=$ $-0.2 \mathrm{eV}$, while the other hopping amplitudes on the $\mathrm{Ti}$ triangle are of absolute value $\leq 0.1 \mathrm{eV}$. Non-surprisingly, this marks Ti2,3 as more strongly coupled, thus possibly

\begin{tabular}{|c|c|c|c|c|c|c|c|}
\hline site & orbital & $\varepsilon_{\mathrm{CF}}$ & $\left|e_{g}^{\prime}(2)\right\rangle$ & $\left|e_{g}^{\prime}(1)\right\rangle$ & $\left|a_{1 g}\right\rangle$ & $\left|e_{g}(1)\right\rangle$ & $\left|e_{g}(2)\right\rangle$ \\
\hline \multirow{3}{*}{ Ti1 } & $\left|\psi_{1}\right\rangle$ & 1102 & 0.035 & -0.999 & 0.000 & 0.000 & -0.002 \\
\hline & $\left|\psi_{2}\right\rangle$ & 698 & 0.000 & 0.000 & -0.147 & -0.006 & 0.990 \\
\hline & $\left|\psi_{3}\right\rangle$ & 1023 & 0.000 & 0.001 & 0.990 & -0.003 & 0.147 \\
\hline \multirow{3}{*}{ Ti2 } & & 710 & -0.032 & -0.023 & 0.837 & -0.427 & -0.214 \\
\hline & $\left|\varphi_{2}\right\rangle$ & 785 & 0.077 & 0.073 & 0.308 & 0.733 & 0.473 \\
\hline & $\left|\varphi_{3}\right\rangle$ & 992 & -0.759 & -0.642 & -0.221 & 0.096 & 0.058 \\
\hline \multirow{3}{*}{ Ti3 } & $\left|\varphi_{1}^{\prime}\right\rangle$ & 709 & 0.036 & -0.027 & 0.872 & 0.437 & -0.220 \\
\hline & $\left|\varphi_{2}^{\prime}\right\rangle$ & 780 & 0.083 & -0.079 & -0.488 & 0.726 & -0.470 \\
\hline & $\left|\varphi_{3}^{\prime}\right\rangle$ & 992 & 0.758 & -0.641 & 0.018 & -0.103 & 0.063 \\
\hline
\end{tabular}

TABLE III. Effective $\mathrm{V}_{\mathrm{O}}$-induced orbitals on the three nearby $\mathrm{Ti}$ sites in the rutile structure, with their respective crystalfield level $\varepsilon_{\mathrm{CF}}$ (in meV), written in terms of linear combinations of the original $\left(t_{2 g}, e_{g}\right)$ functions.

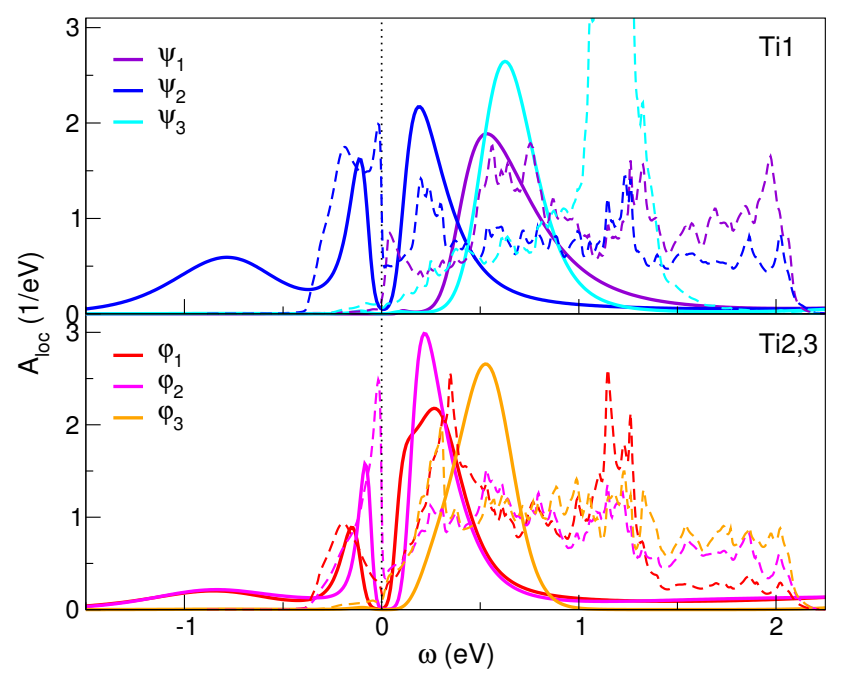

FIG. 5. (color online) Local spectral information for rutile$\mathrm{TiO}_{1.9}$ on Ti1 and Ti2,3 (cf. Fig. 3) at $T=290 \mathrm{~K}$. Dashed lines correspond to the GGA result, respectively.

prone to singlet/triplet formation.

Figure 5 provides the spectra on Ti1-3, which are expected to display (partly) $\mathrm{Ti}^{3+}$ character. Note that although all Ti sites in the supercell contribute to the full correlated subspace, electron occupation on the remaining Ti sites is very small. The filling on the Ti sites farther away from $V_{O}$ is also not significantly raised with temperature. The effective orbitals on Ti2 and Ti3 are nearly equivalent by symmetry and behave here very similarly, so no site differentiation is needed. Of the three correlated orbitals $\left\{\psi_{m}\right\}$ on Ti1, only $\psi_{2}$ has sizable filling. Spurious occupation of $\psi_{1}, \psi_{3}$ in GGA is eliminated by correlations. On the contrary, two orbitals, i.e. $\varphi_{1}$ and $\varphi_{2}$ are occupied on Ti2,3. The designation of $\psi_{2}, \varphi_{1}$ and $\varphi_{2}$ is already suggested from their favorable crystal-field levels (cf. Tab. III). In DFT+DMFT the occupations read $\left(n_{\psi_{2}}, n_{\varphi_{1}}, n_{\varphi_{2}}\right)=(0.65,0.29,0.33)$, 


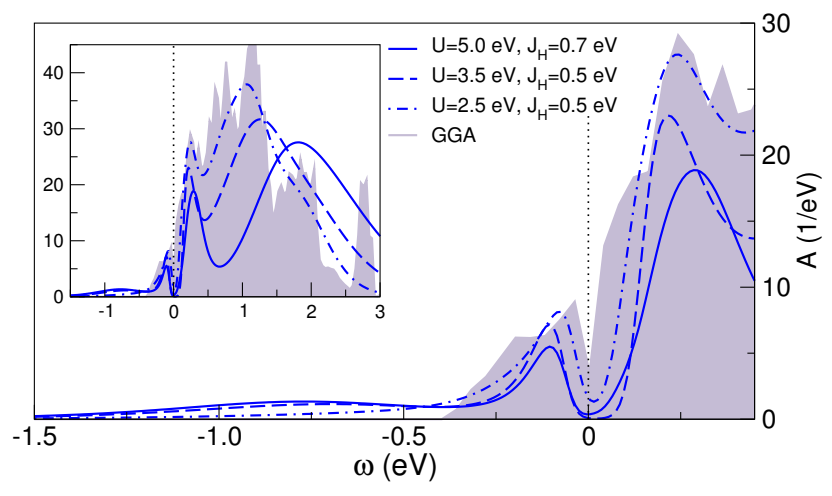

FIG. 6. (color online) Total spectral function for rutile $\mathrm{TiO}_{1.9}$ based on three different sets of local Coulomb interactions at $T=290 \mathrm{~K}$.

thus all three $\mathrm{Ti}$ sites have similar occupation, however $n_{\mathrm{Ti} 1}>n_{\mathrm{Ti} 2,3}$ holds. Since the site-resolved fillings sum up to $n_{V_{\mathrm{O}}}=1.89$ and oxygen is in the $\mathrm{O}^{2-}$ oxidation state in the compound, the defect is described as of being close to the nominal neutral vacancy state $\mathrm{V}_{\mathrm{O}}^{0}$.

In order to check for the influence of the chosen local Coulomb interactions on the physics, we additionally peformed calculations for $U=3.5 \mathrm{eV}$ and $U=2.5 \mathrm{eV}$, both with $J_{\mathrm{H}}=0.5 \mathrm{eV}$. The values $U=3.5 \mathrm{eV}, J_{\mathrm{H}}=0.5 \mathrm{eV}$ were used in a recent DFT+DMFT study of the oxygendeficient $\mathrm{SrTiO}_{3}$ surface $\underline{6}$ There are no qualitative differences between the resulting total spectral functions (cf. Fig. 6) for the sets $\left(U=5 \mathrm{eV}, J_{\mathrm{H}}=0.7 \mathrm{eV}\right)$ and $\left(U=3.5 \mathrm{eV}, J_{\mathrm{H}}=0.5 \mathrm{eV}\right)$, hence the detected physics is rather stable within a reasonable range of local Coulombinteraction parameters. Only at much smaller $U=2.5 \mathrm{eV}$ the semiconducting gap as well as the deeper in-gap state seem to disappear.

It remains to specify the likely mechanism behind the DFT+DMFT finding. The results suggest that the $\mathrm{V}_{\mathrm{O}}$ provides electron doping by initially forming rather shallow states below the original $t_{2 g}$ mainfold. Because of the higher $\mathrm{O}-\mathrm{Ti}$ connectivity and the entangled $\left(e_{g}, t_{2 g}\right)$ defect signature in rutile, both compared to e.g. surface $\left.\mathrm{SrTiO}_{3}, 6\right]$ the coherency of those states is increased and they seemingly develop band-like character. Sizable Coulomb interactions then lead to a Mott criticality, resulting in band renormalization and formation of Hubbard(-like) bands. The latter give rise to the deeplevel in-gap spectra. Importantly, the driving force between the Mott(-like) gap formation is different from a conventional correlated multi-band lattice problem. Since the local states near the $\mathrm{V}_{\mathrm{O}}$ are connected between different $\mathrm{V}_{\mathrm{O}}$ s by fragile hopping paths, mainly the local Coulomb interactions on the $\mathrm{Ti}$ sites distant from the defects are determinative for driving the doped system again insulating. In other words, the 'interstitial' Coulomb-repulsive region between the $\mathrm{V}_{\mathrm{O}}$ s destroys the fragile band-like defect states and localizes the electrons dominantly near the oxygen vacancy.

\section{Excited correlated subspace formed by Ti1, Ti2, Ti3}

Letting only the sites Ti1-3 contribute to the correlated subspace serves to goals. First, it gives access to a possibly different $\mathrm{V}_{\mathrm{O}}$ charging state. Second, it provides a check for our presented mechanism, denoting the Coulomb interactions on $\mathrm{Ti}$ away from $\mathrm{V}_{\mathrm{O}}$ as being mainly to be blamed for the semiconducting character.

In general, the states $\mathrm{V}_{\mathrm{O}}^{0}, \mathrm{~V}_{\mathrm{O}}^{+1}$ and $\mathrm{V}_{\mathrm{O}}^{+2}$ are discussed for oxygen-deficient $\mathrm{TiO}_{2}, \frac{18|22| 2465}{6 h i c h}$ in other works appeared relevant to fit findings of shallow-donor properties and well-localized defect states within a coherent picture. As discussed in section III B 2, the present DFT +DMFT study already provides means to such a coexistence by revealing small-gap as well as deeper in-gap features. Nonetheless, by performing additional calculations within the local-restriced correlated subspace one may learn further details of the $\mathrm{V}_{\mathrm{O}}$ energy-level structure. Note that in principle the local Coulomb interactions also change when the correlated subspace is modified, i.e. in the present scenario should be lowered for the smaller subspace. But for simplicity we keep $U=5 \mathrm{eV}$

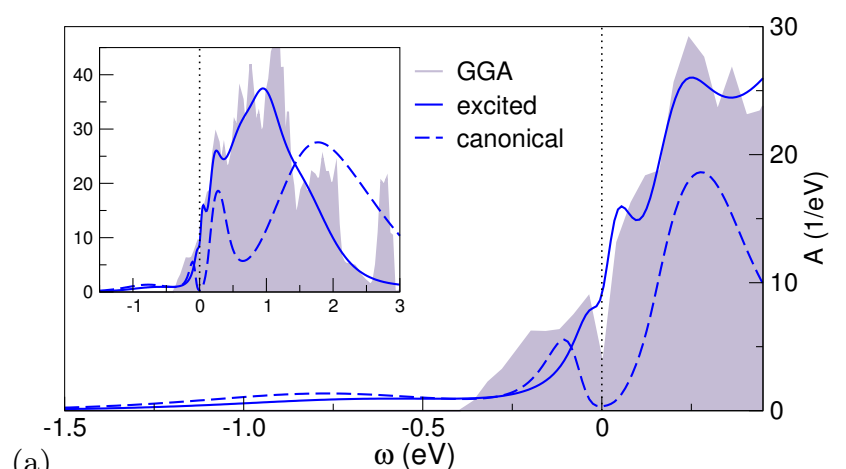

(a)

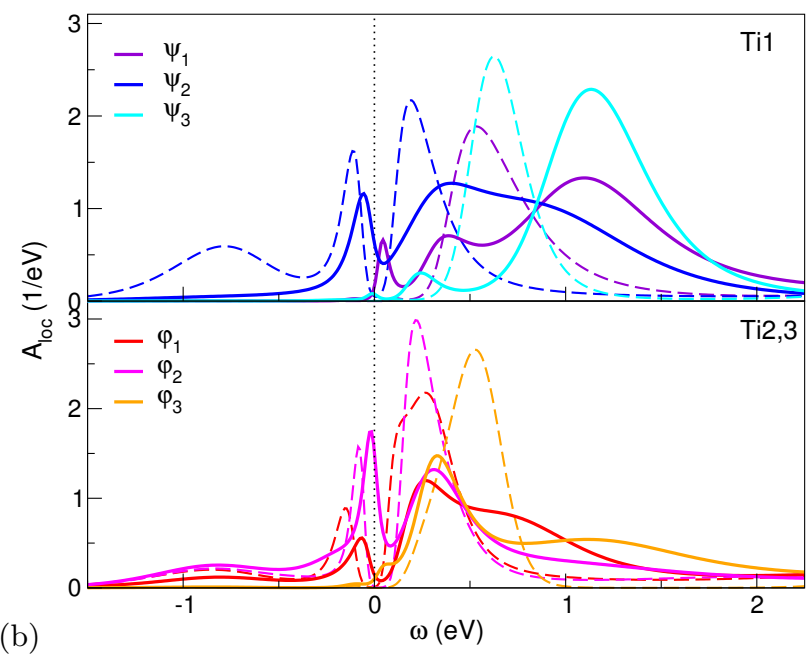

(b)

FIG. 7. (color online) Comparison of spectral information for rutile- $\mathrm{TiO}_{1.9}$ using the canonical and the excited correlated subspace at $T=290 \mathrm{~K}$. (a) Total spectral function. (b) Local spectra for Ti1 and Ti2,3 (cf. Fig. 3) at $T=290 \mathrm{~K}$, full lines: excited, dashed lines: canonical. 
and $J_{\mathrm{H}}=0.7 \mathrm{eV}$ on Ti1-3 to reveal the key features of the excited system.

As expected, a metallic solution results from the calculation, with reduced and shifted incoherent weight at higher energy (see Fig. 7a). This is indeed in favor of our 'interstitial Coulomb' mechanism being relevant for fully localizing electrons near $\mathrm{V}_{\mathrm{O}}$ s. Interestingly, the Ti1-3 sites now display a rather different local filling in the dominant orbitals, namely $\left(n_{\psi_{2}}, n_{\varphi_{1}}, n_{\varphi_{2}}\right)=$ $(0.24,0.19,0.43)$, and a total filling of these sites amounting to $n_{V_{\mathrm{O}}}=1.56$. This means that in the excited scheme not only the total filling of $\mathrm{Ti}$ close to $\mathrm{V}_{\mathrm{O}}$ is substantially reduced compared to the canonical scheme, but also the local filling symmetry is different. Now the Ti2,3 sites are majorly occupied and the Ti1 site plays the weaker role, also visualized by plotting the local spectral functions in Fig. 7b. The high-energy, incoherent and stronglylocalized part of the spectra is now dominantly carried by states on Ti2,3. It appears that in the excited-system calculation, localized charge on Ti1 has been partially transfered to itinerant states.

These findings are indeed reminiscent of the identification of different $\mathrm{V}_{\mathrm{O}}$ charging states in $\mathrm{DFT}+\mathrm{U}$ and/or DFT hybrid-functional studies 1822124 Also the characterization of two different site-orbital levels has been reported before, yet usually by invoking explicit magnetic ordering. Our two detected charging states with $n_{V_{\mathrm{O}}}^{\text {can }}=1.89$, reading $\mathrm{V}_{\mathrm{O}}^{+0.11}$, and $n_{V_{\mathrm{O}}}^{\mathrm{ex}}=1.56$, reading $\mathrm{V}_{\mathrm{O}}^{+0.44}$, do not very strongly deviate from the neutralvacancy case. However note that highly-oxidized states like $\mathrm{V}_{\mathrm{O}}^{+1} / \mathrm{V}_{\mathrm{O}}^{+2}$ are usually found in the presence of additional trivalent substitutional impurities on the Ti site, such as $\mathrm{Fe}^{3+}$ and $\mathrm{Cr}^{3+} ! 65$ An established theoretical picture ${ }^{18}$ describes the vacancy-defect state as a bound object consisting of $\mathrm{V}_{\mathrm{O}}^{2+}$ plus two polarons, rendering it charge neutral again. In principle one may try to interprete our results also along such lines, since the present filling scenario with Hubbard-like high-energy spectral parts point to a local $S=1$ spin in the paramagnetic material. On the other hand, as discussed, we do not have to invoke the polaron picture on clear grounds to account for the coexistence of shallow and deep states.

Let us finally note that the overall qualitative picture that we here derived for a single $\mathrm{V}_{\mathrm{O}}$ in rutile $\mathrm{TiO}_{2}$ is believed to be stable against modified structural relaxations, as e.g. provided by a different(larger) supercell. More dilute $\mathrm{V}_{\mathrm{O}}$ cases could still lead to a weakening of the shallow levels in favor of the deep level, with an increase of the semiconducting gap. Within the local-level manifold the subtle details of the energy hierachy might be affected by structural issues. But the general notion of the relevant $\psi_{2}$ on Ti1 as well as $\varphi_{1}$ and $\varphi_{2}$ on Ti2,3 appears robust.

\section{Higher oxygen-vacancy concentration: $\mathrm{Ti}_{3} \mathrm{O}_{5}$}

\section{Spectral properties and total energies}

We now shift attention to the problem of oxygen vacancies at higher concentration. In order to gain insight in the properties of $\mathrm{V}_{\mathrm{O}} \mathrm{s}$ in a designated ordered limit, we study a specific Magnéli compound at $\mathrm{Ti}_{3} \mathrm{O}_{5}$ stoichiometry. There is strong interest in the various Magnéli phases at $\mathrm{Ti}_{3} \mathrm{O}_{5}, \frac{12 \mid 262766}{68}$ since e.g. photoreversible phase transitions occur at room temperature. But as we are interested in the main effect of vacancy ordering, we focus on a single allotrop, the so-called $\gamma$ phase. The underlying crystal structure has monoclinic symmetry and can be stabilized at room temperature. $\frac{66}{6}$

Described in simple terms, whereas perovskite $\mathrm{SrTiO}_{3}$ has corner-sharing $\mathrm{TiO}_{6}$ octhedra and rutile $\mathrm{TiO}_{2}$ an elementary alternation of corner- and face-sharing octahedra, the Magnéli phases exhibit more complicated arrangement of those two octahedra-sharing types to accomodate a desired stoichiometry (see Fig. 83). This may be interpreted as an ordering of vacancies, however importantly, contrary to e.g. our $\mathrm{TiO}_{1.9}$ structure, there is no $\mathrm{V}_{\mathrm{O}}$-induced 'destruction' of local $\mathrm{TiO}_{6}$ octhedra. In terms of a formal oxygen deficiency $\delta$, the given $\mathrm{Ti}_{3} \mathrm{O}_{5}$

(a)

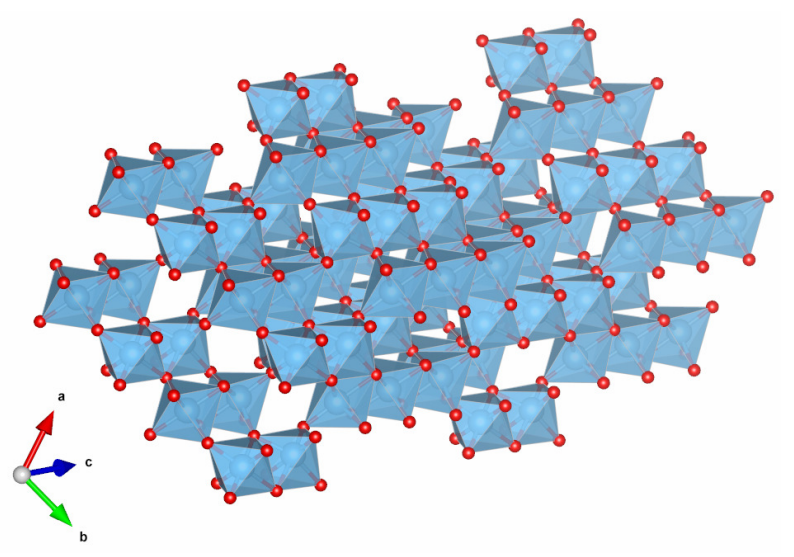

(b)
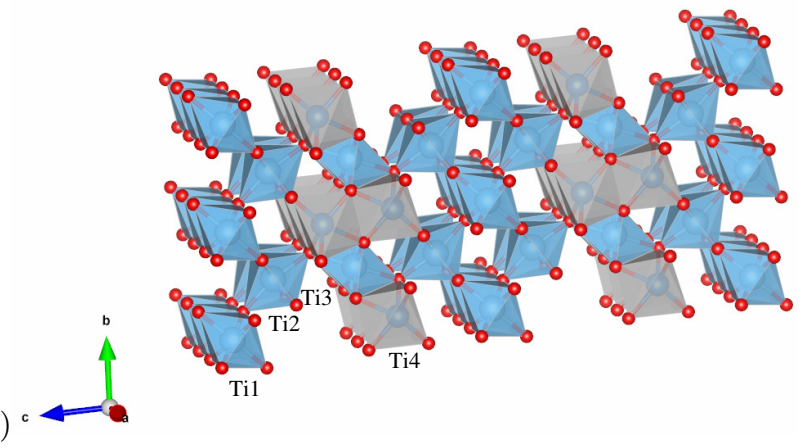

FIG. 8. (color online) Illustration of (a) the $\gamma-\mathrm{Ti}_{3} \mathrm{O}_{5}$ structure and (b) the defect-rutile $\mathrm{Ti}_{3} \mathrm{O}_{5}$ structure, both with $\mathrm{Ti}$ (large blue/grey) and $\mathrm{O}$ (small red/dark) atoms. In (b), the labels Ti1-4 mark symmetry-inequivalent Ti sites, with grey polyhedra surrounding the 5 -fold coordinated Ti4 sites. 


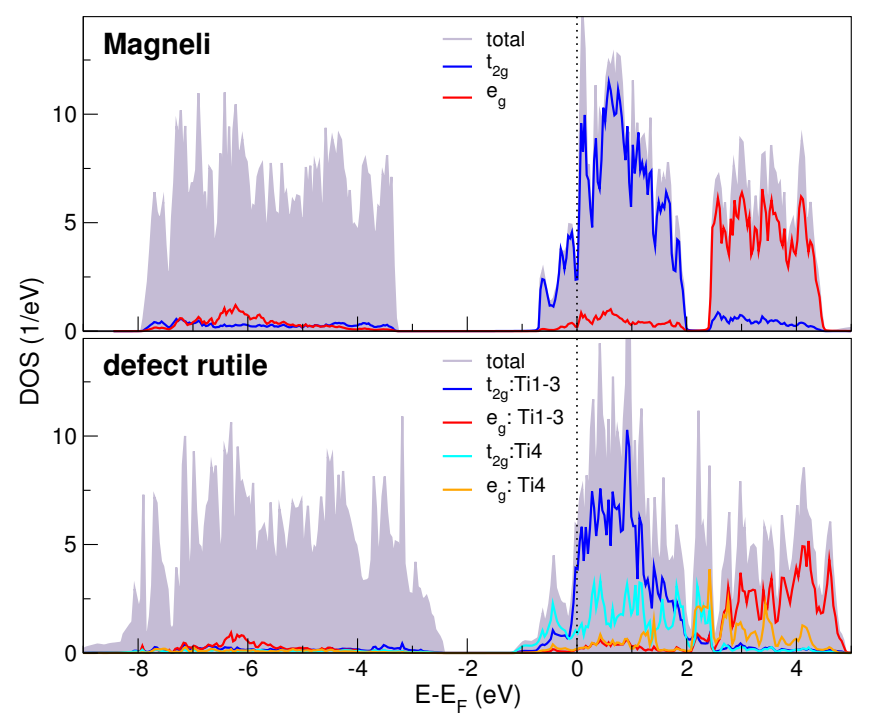

FIG. 9. (color online) Comparison of the GGA density of states of $\gamma-\mathrm{Ti}_{3} \mathrm{O}_{5}$ (top) and defect-rutile $\mathrm{Ti}_{3} \mathrm{O}_{5}$ (bottom), in view of $\left(t_{2 g}, e_{g}\right)$ contributions. The labeling Ti1-3 and Ti4 referes to the sites marked in Fig. 8 p.

stoichiometry amounts nonetheless to $\delta=0.33$, i.e. the compound would correspond to $\mathrm{TiO}_{1.67}$ with vacancy concentration $c=0.167$.

In order to compare the electronic characteristics of the optimal-ordered Magnéli structure with $\mathrm{V}_{\mathrm{O}} \mathrm{s}$ in rutile, we in addition performed calculations for defect rutile with stoichiometrty $\mathrm{Ti}_{3} \mathrm{O}_{5}$. The corresponding crystal structure (cf. Fig. 8b) was determined by energy minimization of oxygen-vacancy arrangements at the desired composition by making use of the cluster-expansion technique (see Ref. 10 for details). In this defect-rutile structure, part of the $\mathrm{TiO}_{6}$ are indeed 'damaged', resulting in selected fivefold-O coordinated $\mathrm{Ti}$ sites, here designated as Ti4. A total-energy comparison on the GGA level, as expected, clearly favors the Magnéli structure. The more clever restoration of ideal 6-fold-oxygen coordination around $\mathrm{Ti}$ is appreciated by a substantial $\sim 0.42 \mathrm{eV}$ per Ti atom against defect rutile.

Figure 9 documents the differences in the GGA density of states in terms of the partitioning in $\mathrm{Ti}\left(t_{2 g}, e_{g}\right)$ like contributions. Based on the robust $\mathrm{TiO}_{6}$-octahedral structuring of the Magnéli phase, the electronic structure of $\gamma-\mathrm{Ti}_{3} \mathrm{O}_{5}$ shows a clear distinction into those $3 d$ submanifolds. This is partly also true for defect-rutile $\mathrm{Ti}_{3} \mathrm{O}_{5}$, yet the $\left(t_{2 g}, e_{g}\right)$ states from the $\mathrm{TiO}_{5}$ polyhedra show there more substantial overlap in energy. In fact, the small $t_{2 g}-e_{g}$ gap region above $2 \mathrm{eV}$ in $\gamma-\mathrm{Ti}_{3} \mathrm{O}_{5}$ just becomes filled by such states in the defect-rutile structure.

The total spectral functions obtained within DFT+DMFT are plotted in Fig. 10. Both systems remain metallic with including many-body correlations. A lower Hubbard band located at $\sim-1.1 \mathrm{eV}$ and renormalization at low energy are identified for the Magnéli

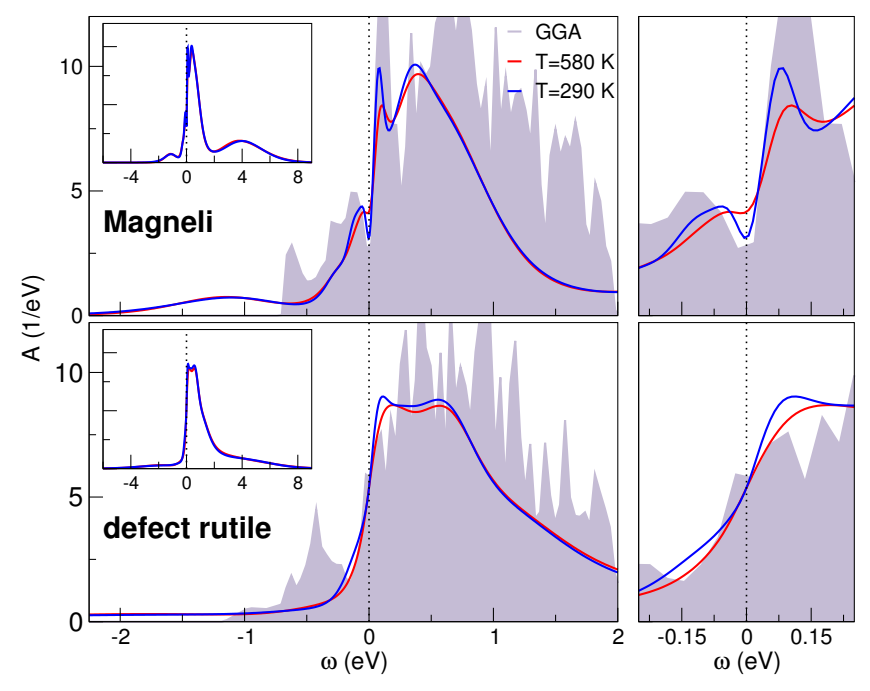

FIG. 10. (color online) Comparison of the correlated total spectral function of $\gamma-\mathrm{Ti}_{3} \mathrm{O}_{5}$ (top) and defect-rutile $\mathrm{Ti}_{3} \mathrm{O}_{5}$ (bottom) at two different temperatures.

phase. This marks the system as a seemingly 'textbook' correlated material, with coherent renormalized quasiparticles at low energy and incoherent Hubbard bands at higher energies. Already on the GGA level there is a small spectral dip at the Fermi level, verified also with correlations at lower temperature. Note that the Hubbard band is of course nearly exclusively formed by $t_{2 g}$-like states. Satellite structures of $t_{2 g}$ kind have recently been detected in a hard x-ray photoelectron spectroscopy study of the structurally different $\beta-\mathrm{Ti}_{3} \mathrm{O}_{5}$ and $\lambda-\mathrm{Ti}_{3} \mathrm{O}_{5}$ Magnéli compounds. 69

Though the same total filling scenario holds for the defect-rutile phase, the characterization with electronic correlations appears more subtle. First, in view of the semiconducting defect-rutile state discussed in section III B 2, the metallic response at higher $\mathrm{V}_{\mathrm{O}}$ concentration is not that surprising. The fragile hopping paths between $\mathrm{V}_{\mathrm{O}}$-near electronic states become increasingly robust with growing concentration of vacancies, such that eventually Coulomb repulsion is not anymore capable of establishing a charge-gapped material. In the low-energy spectrum there is some renormalization due to correlations, but not of significant kind. On a first glance, the spectral function looks furthermore rather monotonic in the occupied part, yet importantly, not necessarily implying that correlations are weak. Because the comparison to the GGA results shows, there is significant spectralweight transfer to energies far away from the Fermi level, too. However this transfer does not give rise to an obvious lower Hubbard-band peak, but is broadly distributed over a wider energy range (see inset of Fig. 10). This finding is somewhat reminiscent of obvervations made in resonant-photoemission experiments on electron-doped $\mathrm{SrTiO}_{3}{ }^{70}$ There, broader in-gap weight was assigned to the increased relevance of $\mathrm{Ti}(3 d)-\mathrm{O}(2 p)$ hybridization. Since the $e_{g}$ character, which is more strongly hybridized 
with $\mathrm{O}(2 p)$ than $t_{2 g}$, plays a more prominent role in the defect-rutile case, the present result could possibly point to a similar interpretation. On the other hand, a recent GW+DMFT study suggests the possibility of diminished satellite peaks in some correlated compounds, as well as a reinterpretation of their original character ${ }^{71}$

The total-energy difference between both $\mathrm{Ti}_{3} \mathrm{O}_{5}$ structural types is even slightly increasing with including many-body correlations. At room temperature, the Magnéli phase is favored by $\sim 0.46 \mathrm{eV}$ per Ti atom.

\section{Charge disproportionation between Ti sites}

So far we did not comment on the local occupations of the respective Ti sites in $\gamma$ - and defect-rutile $\mathrm{Ti}_{3} \mathrm{O}_{5}$. Since the nominal oxidation state at that stoichiometry amounts to $\mathrm{Ti}^{3.33+}$, charge fluctuations are expected to be more relevant than in many other oxides with nominal integer Ti valence. In fact, charge ordering in connection with a metal-insulator transition is commonly discussed for various Magnéli phases, especially for the $\mathrm{Ti}_{4} \mathrm{O}_{7}$ compound. 25

The oxygen deficiency quite naturally introduces symmetry-inequivalent $\mathrm{Ti}$ sites with potentially different electron occupation. Figure $8 \mathrm{~b}$ shows four different $\mathrm{Ti}$ sites with especially the Ti4 site surrounded only by five oxygen atoms. The $\gamma-\mathrm{Ti}_{3} \mathrm{O}_{5}$ structure formally has eight Ti sites different by symmetry, which were also differently treated in our calculations. In the discussion however, to a very good approximation, four Ti classes may be grouped to a single effective class, respectively, since the local-orbital structure only marginally differs. Figure 11 displays the present Magnéli structure in that effective two-Ti-sublattice picturing. An obvious pattern is derived therefrom. The $\mathrm{TiO}_{6}$ octahedra of $\mathrm{Ti}$ sites within a given sublattice are corner sharing, whereas the octahedra are edge sharing between both sublattices. Therefore the intra-sublattice Ti-Ti distance is about $0.5 \AA$ longer than the inter-sublattice pair distance. Both effective sublattices are not of equal-site size, since the Ti2 sublattice (dark-blue octahedra in Fig. 11) covers twice as many Ti sites as the Ti1 sublattice (light-blue octahedra in Fig. 11). Note that the Ti1 octahedra mark the middle of the stoichiometric undisturbed $\mathrm{TiO}_{2}$ rutile slabs, which consist of three octahedra in the rutile (001) direction and are interrupted by a (121) $\mathrm{V}_{\mathrm{O}}$ defect plane.

Tables $\mathrm{IV}$ and $\mathrm{V}$ provide the $\mathrm{Ti}(3 d)$ occupations for both considered $\mathrm{Ti}_{3} \mathrm{O}_{5}$ structures. Nonsurprisingly in defect rutile, the charge on the Ti4 sites with 5 -fold oxygen coordination is largest, marking that site with a $\mathrm{Ti}^{2.8+}$ oxidation state. The nearby Ti3 sites are still close to $\mathrm{Ti}^{3+}$, while Ti1-2 are closer to $\mathrm{Ti}^{4+}$. There are no dramatic differences between the numbers based on GGA and those from DFT+DMFT, only the occupation of Ti4 is still somewhat higher with correlations.

The Magnéli phase $\gamma-\mathrm{Ti}_{3} \mathrm{O}_{5}$ has a more subtle Ti ordering. The geometrical constraints of keeping the $\mathrm{TiO}_{6}$

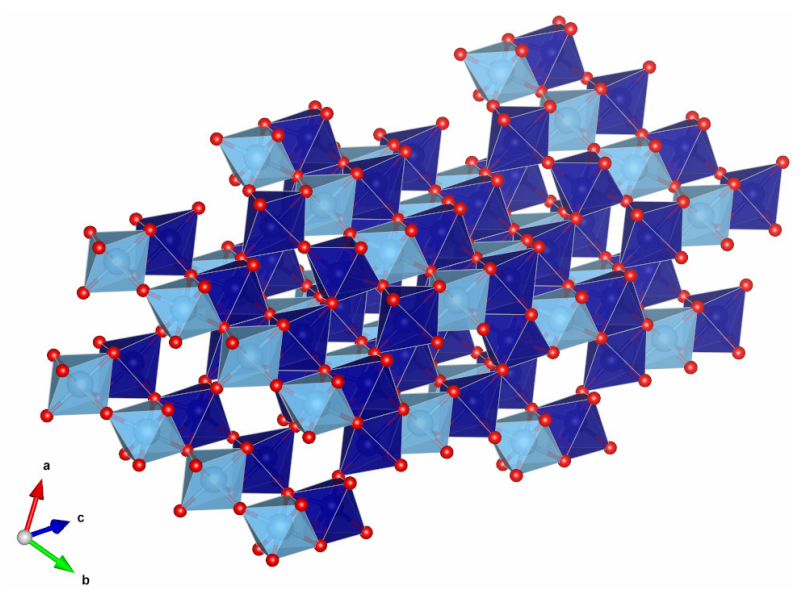

FIG. 11. (color online) Effective two-Ti-sublattice representation of $\gamma-\mathrm{Ti}_{3} \mathrm{O}_{5}$ (see text). Light(Dark) blue octahedra correspond to $\mathrm{Ti}$ sites on sublattice $1(2)$.

\begin{tabular}{l|cc}
\hline \hline & GGA & DFT + DMFT $(U=5 \mathrm{eV})$ \\
\hline Ti1 & 0.22 & 0.20 \\
Ti2 & 0.26 & 0.24 \\
Ti3 & 0.90 & 0.86 \\
Ti4 & 1.15 & 1.23 \\
\hline \hline
\end{tabular}

TABLE IV. Comparison of the $3 d$ filling of the different Ti sites in defect-rutile $\mathrm{Ti}_{3} \mathrm{O}_{5}$ (see Fig. $8 \mathrm{p}$ for Ti labeling). The DFT+DMFT data is taken at $T=290 \mathrm{~K}$.

\begin{tabular}{c|ccc}
\hline \hline & GGA & \multicolumn{2}{|c}{ DFT+DMFT } \\
\hline & & $U=5 \mathrm{eV}$ & $U=8 \mathrm{eV}$ \\
Ti1 & 0.740 & 0.752 & 0.766 \\
Ti2 & 0.630 & 0.624 & 0.617 \\
\hline \hline
\end{tabular}

TABLE V. Comparison of the $3 d$ filling on the two effective sublattices in $\gamma-\mathrm{Ti}_{3} \mathrm{O}_{5}$ (see Fig. 11 for Ti-sublattice labeling). The DFT+DMFT data is taken at $T=290 \mathrm{~K}$.

octahedra in line with the nominal $\mathrm{Ti}^{3.33+}$ state leads to slightly different charge states on both effective sublattices. The smaller Ti1 sublattice carries already on the GGA level more charge, with a disproportionation $\rho($ Ti1 $)-\rho($ Ti2 $)=0.11 \mathrm{e}^{-}$. Electronic correlations increase this charge splitting only by small amounts, even when invoking a rather large Hubbard $U=8 \mathrm{eV}$. Importantly, the system remains always metallic. Also because a seemingly straightforward $\mathrm{Ti}^{3+} / \mathrm{Ti}^{4+}$ splitting, as in principle possible in the $\mathrm{Ti}_{4} \mathrm{O}_{7}$ Magnéli system with a nominal $\mathrm{Ti}^{3.5+}$ oxidation state, is not an obvious option. Note however that there is also a metal-insulator transition reported for Magnéli $\mathrm{Ti}_{3} \mathrm{O}_{5}, \frac{27}{}$ but from $\gamma-\mathrm{Ti}_{3} \mathrm{O}_{5}$ to $\delta-\mathrm{Ti}_{3} \mathrm{O}_{5}$ upon lowering temperature. Thus further structural changes are in addition indispensable to allow for insulating behavior. 


\section{Summary and discussion}

We presented a detailed first-principles many-body investigation of the effect of oxygen vacancies in rutile $\mathrm{TiO}_{2}$, both in the lower- and the higher-concentration regime. In the former case, the DFT+DMFT results directly provide the three key ingredients known from experiment for $\mathrm{TiO}_{2-\delta}$, namely semiconducting behavior, shallow levels as well as deep levels. Our deep-level (or in-gap) positioning is in excellent agreement with results from various experimental studies. Thereby the manybody perspective provides a different viewpoint on the longstanding discussion of deep vs. shallow levels for oxygen-deficient $\mathrm{TiO}_{2}$. Here, both levels are connected in the many-body sense, similar as a lower Hubbard band and a renormalized quasiparticle state in a conventional moderately correlated metal. Still the correlationinduced gap opening in the band-like initial shallow levels is not of standard Mott type. Key to the electron localization at low $\mathrm{V}_{\mathrm{O}}$ concentration is the Coulomb-repulsion region between defects, that blocks the fragile hopping paths. Therefore, the resulting charge gap is rather small and does not scale with $U$ in a conventional way.

By invoking an excited correlated subspace we brought the semiconducting solution into the transport regime, therewith showing that indeed the Coulomb interactions on the Ti sites distant from the oxygen vacancies dominantly control the competition between itinerancy and localization. Moreover, the localized states at the $\mathrm{V}_{\mathrm{O}}$ display a different occupation and energy state upon excitation. This connects our study to other (staticcorrelation) theory work for $\mathrm{TiO}_{2-\delta}$, where e.g. different $\mathrm{V}_{\mathrm{O}}$ charge states and polaron formation are discussed! 16 24

In comparison to oxygen-deficient $\mathrm{SrTiO}_{3}$, the defectrutile problem differs in two crucial point. First, the defect structure with a $\mathrm{V}_{\mathrm{O}}$ in nearest-neighbor distance to three Ti sites, contrary to two Ti sites in the perovskite structure, fosters a modified oxygen-vacancy impact. The higher connectivity with the defect gives way to the formation of more band-like impurity states on the single-electron level. Second, the higher entanglement between $t_{2 g}$ and $e_{g}$ states lifts the electron dichotomy observed on the $\mathrm{SrTiO}_{3-\delta}$ surface $\frac{6}{6}$ suppressing both, a decoupled $t_{2 g}$ band formation and a rather disconnect straightforward $e_{g}$ deep-level formation. Therefore at small $\mathrm{V}_{\mathrm{O}}$ concentrations, rutile $\mathrm{TiO}_{2}$ remains insulating (or semiconducting) due to correlation-induced gap opening in $\left(t_{2 g}, e_{g}\right)$-entangled band-like impurity levels. Note in this context however that the very correlation details of bulk $\mathrm{SrTiO}_{3-\delta}$ still need to be investigated by similar means

In the higher $\mathrm{V}_{\mathrm{O}}$-concentration regime we focussed on the $\mathrm{Ti}_{3} \mathrm{O}_{5}$ stoichiometry, studying the metallic Magnéli $\gamma$-phase as well as a theory-derived metallic defect-rutile phase. The interplay of Ti coordination and $\left(t_{2 g}, e_{g}\right)$ electronic structure properties were discussed and therefrom the differences in total energy and in the spectral features explained. While $\gamma-\mathrm{Ti}_{3} \mathrm{O}_{5}$ turns out as a 'textbook'like correlated metal, the defect-rutile version displays spectral-weight transfers to higher energies in a much broader (incoherent) fashion. Charge disproportionation is a natural by-product of the $\mathrm{V}_{\mathrm{O}}$ ordering in rutile-based $\mathrm{TiO}_{2}$ (which formally includes the Magnéli phases) ${ }^{15}$ and is already captured on the Kohn-Sham level. Electronic correlations beyond the latter provide at least for $\mathrm{Ti}_{3} \mathrm{O}_{5}$ only minor changes to the Ti charging states.

Although in direct comparison, defect-rutile $\mathrm{Ti}_{3} \mathrm{O}_{5}$ is energetically rather unfavorable compared to the Magnéli phase, its discussion is nonetheless revelant in view of engineering memristive processes and devices. Since there in $\mathrm{TiO}_{2}$, transport of $\mathrm{V}_{\mathrm{O}}$ and electrons when starting from the rutile structure is a possible technological aspect. In fact, in realistic close-to-device-like $\mathrm{TiO}_{2}$ materials, the formation of defect-rutile and Magnéli $\operatorname{Ti}_{n} \mathrm{O}_{2 n-1}$ phases may happen in parallel within a given materials system.9 Thus understanding the differences between both structural types on a basic level is of crucial importance. In the long run, the present study shall contribute to pave the road for elucidating further engineering options in the interplay between the transport of oxygen vacancies and electrons.

It would also be highly interesting to extent the present investigation to the rutile $\mathrm{VO}_{2}$ system. As the vanadium ion in the +4 oxdidation state has $3 d^{1}$ occupation, it is known that already the stoichiometric system is prone to strong correlation physics, ${ }^{72} \frac{76}{76}$ possibly giving reason to the hallmark metal-to-insulator transition slightly above room temperature ${ }^{77}$ Recent doping studies of $\mathrm{VO}_{2}$ via oxygen vacancies display opportunities to tune the competition between the metallic and insulating regime. ${ }^{78 / 79}$

Finally coming back to basic features of $\mathrm{V}_{\mathrm{O}} \mathrm{s}$ in transition-metal oxides, our examination challenges the simplest views on in-gap states, namely the weakcoupling defect-level and the strong-coupling Hubbardband paradigm. Albeit one encounters features of both original mechanisms in oxygen-deficient $\mathrm{TiO}_{2}$, as in related systems such as $\mathrm{SrTiO}_{3-\delta}$ or the $\mathrm{LaAlO}_{3} / \mathrm{SrTiO}_{3}$ interface, a unique and well-defined picture describing the general nature of defects in transition-metal oxides is still missing. Crystal-field effects, renormalizations, Hubbard-band formation, lifetime effects, $p$ - $d$ hybridization, screening, charge transfer, polaron formation, etc. are potentially part of this demanding physics. Thoughts trying to put the problem in an adequate model setting have been put forward from several perspectives, e.g. Ref. 80. The future task is to cast those into a sound and solid materials-dependent picture.

\section{ACKNOWLEDGMENTS}

We gratefully acknowledge financial upport from the German Science Foundation (DFG) via SFB986 and through FOR1346. Computations were performed at the University of Hamburg and the JURECA Cluster of the 
Jülich Supercomputing Centre (JSC) under project num- $\quad$ ber hhh08.

1 W. Luo, W. Duan, S. G. Louie, and M. L. Cohen, Phys. Rev. B 70, 214109 (2004).

2 N. Pavlenko, T. Kopp, E. Y. Tsymbal, J. Mannhart, and G. A. Sawatzky, Phys. Rev. B 86, 064431 (2012).

3 C. Mitra, C. Lin, J. Robertson, and A. A. Demkov, Phys. Rev. B 86, 155105 (2012).

4 J. Shen, H. Lee, R. Valentí, and H. O. Jeschke, Phys. Rev. B 86, 195119 (2012).

${ }^{5}$ F. Lechermann, L. Boehnke, D. Grieger, and C. Piefke, Phys. Rev. B 90, 085125 (2014).

${ }^{6}$ F. Lechermann, H. O. Jeschke, A. J. Kim, S. Backes, and R. Valentí, Phys. Rev. B 93, 121103(R) (2016).

7 M. Altmeyer, H. O. Jeschke, O. Hijano-Cubelos, C. Martins, F. Lechermann, K. Koepernik, A. Santander-Syro, M. J. Rozenberg, R. Valentí, and M. Gabay, Phys. Rev. Lett. 116, 157203 (2016).

8 J. J. Yang, D. B. Strukov, and D. R. Stewart, Nat. Nanotechnol. 8, 13 (2013).

9 D.-H. Kwon, K. M. Kim, J. H. Jang, J. M. Jeon, M. H. Lee, G. H. Kim, X.-S. Li, G.-S. Park, B. Lee, S. Han, et al., Nat. Nanotechnol. 5, 148 (2010).

10 W. Heckel, M. Wehlau, S. B. Maisel, T. Frauenheim, J. M. Knaup, and S. Müller, Phys. Rev. B 92, 214104 (2015).

11 M. Behrmann and F. Lechermann, Phys. Rev. B 92, 125148 (2015).

12 S. Åsbrink and A. Magnéli, Acta Cryst. 12, 575 (1959).

13 S. Andersson and L. Jahnberg, Ark. Kemi 21, 413 (1963).

14 L. A. Bursill and B. G. Hyde, Prog. Solid State Chem. 7, 177 (1972).

15 L. Liborio and N. Harrison, Phys. Rev. B 77, 104104 (2008).

16 A. Janotti, J. B. Varley, P. Rinke, N. Umezawa, G. Kresse, and C. G. V. de Walle, Phys. Rev. B 81, 085212 (2010).

17 P. Deák, B. Aradi, and T. Frauenheim, Phys. Rev. B 86, 195206 (2012).

18 A. Janotti, C. Franchini, J. B. Varley, G. Kresse, and C. G. V. de Walle, Phys. Status Solidi RRL 7, 199 (2013).

19 D. Berger, H. Oberhofer, and K. Reuter, Phys. Rev. B 92, 075308 (2015).

20 G. C. Vásquez, S. Z. Karazhanov, D. Maestre, A. Cremades, J. Piqueras, and S. E. Foss, Phys. Rev. B 94, 235209 (2016).

21 G. Mattioli, F. Filippone, P. Alippi, and A. A. Bonapasta, Phys. Rev. B 78, 241201(R) (2008).

22 G. Mattioli, P. Alippi, F. Filippone, R. Caminiti, and A. A. Bonapasta, J. Phys. Chem. C 114, 21694 (2010).

23 B. J. Morgan and G. W. Watson, J. Phys. Chem. C 114, 2321 (2010).

24 C. Lin, D. Shin, and A. A. Demkov, J. Appl. Phys. 117, 225703 (2015).

25 I. Leonov, A. N. Yaresko, V. N. Antonov, U. Schwingenschlögl, V. Eyert, and V. I. Anisimov, J. Phys.: Condens. Matter 18, 10955 (2006).

26 A. Padilha, J. M. Osorio-Guillén, A. R. Rocha, and G. M. Dalpian, Phys. Rev. B 90, 035213 (2014).

27 K. Tanaka, T. Nasu, Y. Miyamoto, N. Ozaki, S. Tanaka, T. Nagata, F. Hakoe, M. Yoshikiyo, K. Nakagawa, Y. Umeta, et al., Cryst. Growth Des. 15, 653 (2015).
28 D. A. H. Hanaor and C. C. Sorrell, J. Mater. Sci. 46, 855 (2011).

29 J. P. Perdew, A. Ruzsinszky, G. I. Csonka, O. A. Vydrov, G. E. Scuseria, L. A. Constantin, X. Zhou, and K. Burke, Phys. Rev. Lett. 100, 136406 (2008).

${ }^{30}$ G. Kresse and J. Hafner, Phys. Rev. B 47, 558 (1993).

31 G. Kresse and J. Hafner, Phys. Rev. B 49, 14251 (1994).

32 G. Kresse and J. Furthmüller, Phys. Rev. B 54, 11169 (1996).

33 G. Kresse and J. Furthmüller, Computational Materials Science 6, 15 (1996).

34 S. Y. Savrasov, G. Kotliar, and E. Abrahams, Nature 410, 793 (2001).

35 L. V. Pourovskii, B. Amadon, S. Biermann, and A. Georges, Phys. Rev. B 76, 235101 (2007).

36 D. Grieger, C. Piefke, O. E. Peil, and F. Lechermann, Phys. Rev. B 86, 155121 (2012).

37 S. G. Louie, K. M. Ho, and M. L. Cohen, Phys. Rev. B 19, 1774 (1979).

38 B. Meyer, C. Elsässer, F. Lechermann, and M. Fähnle, Fortran 90 program for mixed-basis-pseudopotential calculations for crystals.

39 A. N. Rubtsov, V. V. Savkin, and A. I. Lichtenstein, Phys. Rev. B 72, 035122 (2005).

40 P. Werner, A. Comanac, L. de' Medici, M. Troyer, and A. J. Millis, Phys. Rev. Lett. 97, 076405 (2006).

41 O. Parcollet, M. Ferrero, T. Ayral, H. Hafermann, I. Krivenko, L. Messio, and P. Seth, Comput. Phys. Commun. 196, 398 (2015).

42 P. Seth, I. Krivenko, M. Ferrero, and O. Parcollet, Comput. Phys. Commun. 200, 274 (2016).

43 J. P. Perdew, K. Burke, and M. Ernzerhof, Phys. Rev. Lett. 77, 3865 (1996).

44 B. Amadon, F. Lechermann, A. Georges, F. Jollet, T. O. Wehling, and A. I. Lichtenstein, Phys. Rev. B 77, 205112 (2008).

45 V. I. Anisimov, D. E. Kondakov, A. V. Kozhevnikov, I. A. Nekrasov, Z. V. Pchelkina, J. W. Allen, S.-K. Mo, H.-D. Kim, P. Metcalf, S. Suga, et al., Phys. Rev. B 71, 125119 (2005).

46 M. Aichhorn, L. Pourovskii, V. Vildosola, M. Ferrero, O. Parcollet, T. Miyake, A. Georges, and S. Biermann, Phys. Rev. B 80, 085101 (2009).

47 K. Haule, C.-H. Yee, and K. Kim, Phys. Rev. B 81, 195107 (2010).

48 M. Karolak, T. O. Wehling, F. Lechermann, and A. I. Lichtenstein, J. Phys.: Condens. Matter 23, 085601 (2011).

49 T. Mizokawa and A. Fujimori, Phys. Rev. B 51, 12880R (1995).

50 E. Pavarini, S. Biermann, A. Poteryaev, A. I. Lichtenstein, A. Georges, and O. K. Andersen, Phys. Rev. Lett. 92, 176403 (2004).

51 S. Okamoto, A. J. Millis, and N. A. Spaldin, Phys. Rev. Lett. 97, 056802 (2006).

52 F. Lechermann and M. Obermeyer, New J. Phys. 17, 043026 (2015).

53 V. I. Anisimov, I. V. Solovyev, M. A. Korotin, M. T. 
Czyżyk, and G. A. Sawatzky, Phys. Rev. B 48, 16929 (1993).

54 J. K. Burdett, T. Hughbanks, G. J. Miller, J. W. RichardsonJr., and J. V. Smith, J. Am. Chem. Soc. 109, 3639 (1987).

55 D. C. Cronemeyer and M. A. Gilleo, Phys. Rev. 82, 975 (1951).

56 D. C. Cronemeyer, Phys. Rev. 87, 876 (1952).

57 J. Pascual, J. Camassel, and H. Mathieu, Phys. Rev. B 18, 5606 (1978).

58 J. C. Parker and R. W. Siegel, Appl. Phys. Lett. 57, 943 (1990).

59 A. G. Thomas, W. R. Flavell, A. K. Mallick, A. R. Kumarasinghe, D. Tsoutsou, N. Khan, C. Chatwin, S. Rayner, G. C. Smith, R. L. Stockbauer, et al., Phys. Rev. B 75, 035105 (2007).

60 S. Wendt, P. T. Sprunger, E. Lira, G. K. H. Madsen, Z. Li, J. Ø. Hansen, J. Matthiesen, A. Blekinge-Rasmussen, E. Lægsgaard, B. Hammer, et al., Science 320, 1755 (2008).

61 C. M. Yim, C. L. Pang, and G. Thornton, Phys. Rev. Lett. 104, 036806 (2010).

62 T. C. Rödel, F. Fortuna, F. Bertran, M. Gabay, M. J. Rozenberg, A. F. Santander-Syro, , and P. Le Fèvre, Phys. Rev. B 92, 041106(R) (2015).

${ }^{63}$ M. Setvin, C. Franchini, X. Hao, M. Schmid, A. Janotti, M. Kaltak, C. G. V. de Walle, G. Kresse, and U. Diebold, Phys. Rev. Lett. 113, 086402 (2014).

64 E. Yagi, R. R. Hasiguti, and M. Aono, Phys. Rev. B 54, 7945 (1996).

65 S. Yang, L. E. Halliburton, A. Manivannan, P. H. Bunton, D. B. Baker, M. Klemm, S. Horn, and A. Fujishima, Appl.
Phys. Lett. 94, 162114 (2009).

66 S.-H. Hong and S. Åsbrink, Acta Cryst. B 38, 2570 (1982).

${ }_{67}$ M. Onoda, J. Sol. State Chem. 136, 67 (1998).

68 S. Ohkoshi, Y. Tsunobuchi, T. Matsuda, K. Hashimoto, A. Namai, F. Hakoe, and H. Tokoro, Nat. Chem. 2, 539 (2010).

69 K. Kobayashi, M. Taguchi, M. Kobata, K. Tanaka, H. Tokoro, H. Daimon, T. Okane, H. Yamagami, E. Ikenaga, and S. Ohkoshi, Phys. Rev. B 95, 085133 (2017).

70 Y. Ishida, R. Eguchi, M. Matsunami, K. Horiba, M. Taguchi, A. Chainani, Y. Senba, H. Ohashi, H. Ohta, and S. Shin, Phys. Rev. Lett. 100, 056401 (2008).

71 L. Boehnke, F. Nilsson, F. Aryasetiawan, and P. Werner, Phys. Rev. B 94, 201106(R) (2016).

72 A. Zylbersztejn and N. Mott, Phys. Rev. B 11, 4383 (1975).

73 C. Sommers and S. Doniach, Solid State Commun. 28, 133 (1978).

74 T. M. Rice, H. Launois, and J. P. Pouget, Phys. Rev. Lett. 73, 3042 (1994).

75 S. Biermann, A. Poteryaev, A. I. Lichtenstein, and A. Georges, Phys. Rev. Lett. 94, 026404 (2005).

76 O. Nájera, M. Civelli, V. Dobrosavliević, and M. J. Rozenberg, Phys. Rev. B 95, 035113 (2017).

77 F. J. Morin, Phys. Rev. Lett. 3, 34 (1959).

78 J. Jeong, N. Aetukuri, T. Graf, T. D. Schladt, M. G. Samant, and S. S. P. Parkin, Science 339, 1402 (2013).

79 Z. Zhang, F. Zuo, C. Wan, A. Dutta, J. Kim, J. Rensberg, R. Nawrodt, H. Hejin Park, T. J. Larrabee, X. Guan, et al., Phys. Rev. Applied 7, 034008 (2017).

80 F. D. M. Haldane and P. W. Anderson, Phys. Rev. B 13, 2553 (1976). 